\title{
Different long-term characteristics of hydraulic pressure gauges under constant pressure applications
}

\author{
Hiroaki Kajikawa ${ }^{1}$, Tokihiko Kobata ${ }^{1}$ \\ ${ }^{1}$ National Metrology Institute of Japan (NMIJ), AIST, 1-1-1 Umezono, Tsukuba-city, Ibaraki, Japan
}

\section{ABSTRACT}

This article focuses on the long-term drifts of hydraulic pressure gauges, which are constantly subjected to high pressures. A target measurement pressure was consistently applied to pressure gauges for more than two months, and the gauges were occasionally calibrated against a reference pressure balance. The long-term drift has been evaluated based on the variations in calibration values with the elapsed time since the pressure application. We evaluated the long-term drift of three kinds of pressure gauges that are commonly used for calibrations and industrial measurements. The long-term drift differed depending on the type of sensing structure. Although the gauges that had similar sensing structures showed similar drift when the gauges were pressurised due to atmospheric pressure, the amount of the drift depended on each product. In addition, the long-term drift was also affected significantly by the preliminary pressure conditions, even under the same pressure application. Preliminary or ex-post evaluations are necessary for the respective gauges under similar pressure conditions. In practice, the drift of zero-reading is often the main contributory factor of the drift; therefore, the drift can be mitigated by tracing the zero-reading during the pressure applications.

\section{Section: RESEARCH PAPER}

Keywords: Pressure gauge; long-term drift; pressure calibration; pressure standard

Citation: Hiroaki Kajikawa, Tokihiko Kobata, Different long-term characteristics of hydraulic pressure gauges under constant pressure applications, Acta IMEKO, vol. 8, no. 3, article 4, September 2019, identifier: IMEKO-ACTA-08 (2019)-03-04

Editor: Jeerasak Pitakarnnop, NIMT, Thailand

Received August 30, 2018; In final form June 17, 2019; Published September 2019

Copyright: This is an open-access article distributed under the terms of the Creative Commons Attribution 3.0 License, which permits unrestricted use, distribution, and reproduction in any medium, provided the original author and source are credited.

Corresponding author: Hiroaki Kajikawa, e-mail: Kajikawa.hiroaki@aist.go.jp

\section{INTRODUCTION}

Long-term drift is one of the important characteristics of pressure gauges and should be evaluated when the gauges are used as the reference standard for pressure calibrations or the transfer standard for inter-laboratory comparisons [1]-[5]. Pressure gauges used as reference gauges in calibration laboratories are calibrated periodically (once a year in most cases), and the long-term drift is then evaluated and considered as an uncertainty factor. When pressure gauges are used as transfer standards for international comparison or proficiency testing of calibration laboratories, the drift of the transfer standard needs to be corrected to appropriately compare the participants' results. The drift is evaluated by the pilot institute at both the beginning and the end of the circulation of the transfer standard. The reports of the international comparisons include the evaluation of the long-term drift of the transfer standard [6], [7]. In the above cases, pressure gauges of interest are always kept in stable condition, and no pressure is applied except when they are used in measurements. Furthermore, the gauges are used with a fixed procedure according to wellrecognised protocols, such as stepwise calibration cycles. Thus, the long-term drift of these gauges can be appropriately evaluated and corrected by periodic calibrations.

In scientific and industrial fields, however, pressure gauges are used in various environment and pressure conditions. Some pressure gauges are used under constant and stable pressures, and others are under periodically changing pressure conditions. Such pressure gauges may show different long-term characteristics from those that are kept in normal atmospheric conditions most of the time. To obtain reliable pressure values with higher accuracy, long-term drift should be evaluated in similar pressure conditions as the actual usage.

In geophysical research, for example, pressure gauges are used for monitoring seafloor pressures to detect the uplift or subsidence of submarine grounds [8]-[10]. Currently, many precise pressure gauges are installed at seafloors around the Japanese islands and other earthquake zones, known as the Circum-Pan-Pacific Earthquake Belt. The pressure gauges installed at seafloors in the depth of several kilometres are 
Table 1. Specifications of pressure gauges used for experiments of long-term drift under constant pressure.

\begin{tabular}{|c|c|c|c|c|c|}
\hline & Type & Full scale & $\begin{array}{c}\text { Accuracy } \\
\text { (of Full Scale) }\end{array}$ & Manufacture, Model & Applied Pressure \\
\hline PT200-1 & Quartz Bourdon-type pressure gauge & $207 \mathrm{MPa}$ & $0.01 \%$ & Paroscientific, Inc., 9000-30K & $200 \mathrm{MPa}, 100 \mathrm{MPa}$ \\
\hline PT200-2 & Clamped thin-film pressure gauge & $250 \mathrm{MPa}$ & $0.25 \%$ & WIKA, HP-2-S & $200 \mathrm{MPa}, 100 \mathrm{MPa}$ \\
\hline PT200-3 & Foil stain pressure gauge & $200 \mathrm{MPa}$ & $0.10 \%$ & HBM, 1-P3TCP & $200 \mathrm{MPa}, 100 \mathrm{MPa}$ \\
\hline PT70-1 & Quartz Bourdon-type pressure gauge & $70 \mathrm{MPa}$ & $0.01 \%$ & Paroscientific, Inc., 9000-10K & $70 \mathrm{MPa}$ \\
\hline PT70-2 & Clamped thin-film pressure gauge & $100 \mathrm{MPa}$ & $0.25 \%$ & WIKA, S-10 & $70 \mathrm{MPa}$ \\
\hline
\end{tabular}

always subjected to high pressures of several tens of MPa. To detect subtle crustal movements from the pressure data, the effect of the long-term drift of the pressure gauges (as well as other influential factors in the seafloor pressure data such as daily tidal fluctuations and seasonal variations in the sea level) should be evaluated and mitigated. The long-term trends of the pressure gauge outputs have been comprehensively evaluated based on the pressure data obtained during actual seafloor measurements [11]. Laboratory experiments of long-term drift were also conducted for some pressure gauges [12]. Currently, the characteristics of the gauges used at several tens of MPa for months and years are required for this purpose. Laboratory experiments for high-pressure gauges can provide the data without other fluctuating factors, and they clearly show the drift trend that is intrinsic to respective gauges.

In this study, we have evaluated the long-term drift of pressure gauges, which are constantly subjected to high pressures for long periods of time, with the aim of suggesting appropriate methods to evaluate and reduce long-term drift [13]. This paper shows the drifts of three kinds of pressure gauges that are used for pressure calibrations and industrial pressure measurements. The measurement system and evaluation methods are explained in section 2. The long-term drifts of the three kinds of gauges are compared in two experiments in section 3. Practical measures to reduce and mitigate the long-term drift are also discussed in Section 3. Finally, a brief summary is given in section 4 .

\section{EXPERIMENT}

Figure 1 shows the measurement system that has been used for the long-term drift evaluation. Three kinds of pressure gauges have been evaluated in this study: quartz Bourdon-type pressure transducers (Paroscientific, Inc.), foil stain pressure gauges (HBM), and clamped thin-film pressure gauges (WIKA). Table 1 shows the detailed information of the test gauges, including the maximum measurement pressure and accuracy

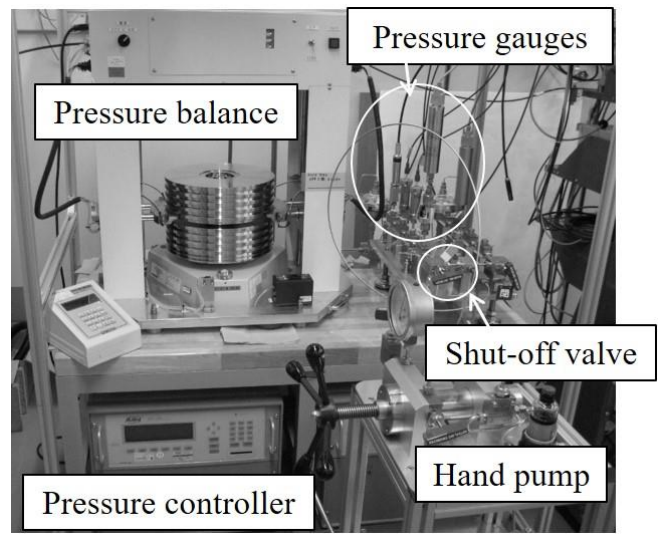

Figure 1. Measurement system for long-term drift evaluation. claimed by the manufacturers. The pressure applied to the test gauges was kept in enclosed tubing by closing a shut-off valve in the system. During the experiment, the pressure was maintained at the target pressure within $1 \mathrm{MPa}$, despite the pressure fluctuation following the variations in the surrounding temperature in the calibration room. An additional valve was also used as a variable volume to adjust the pressure in the enclosed space during pressure application.

During the pressure application, the test gauges were calibrated against a reference pressure balance. First, the system (including the reference pressure balance) was pressurised to the test pressure by using a pressure controller and a hand pump, and then the shut-off valve was opened to apply the pressure controlled with the reference pressure balance. The outputs of the gauges were obtained after an appropriate waiting time for pressure stabilisation. At each test pressure $p$, the deviation of the averaged output of the test gauge $\left(I_{\mathrm{p}}\right)$ from the standard pressure by the reference pressure balance $\left(p_{\mathrm{s}}\right), D I_{\mathrm{p}}$ was calculated as $D I_{\mathrm{p}}=I_{\mathrm{p}}-p_{\mathrm{s}}$. After the measurement, the shut-off valve was closed to maintain the pressure on the test gauges, and the pressure of the reference side was released to atmospheric pressure. The long-term drifts of the pressure gauges were evaluated by tracing the variations in $D I_{\mathrm{p}}$ according to time.

\section{RESULTS AND DISCUSSION}

\subsection{Different long-term drifts under constant pressure}

Figure 2(a) shows the one-year history of the pressure application for three pressure gauges under test: PT200-1,
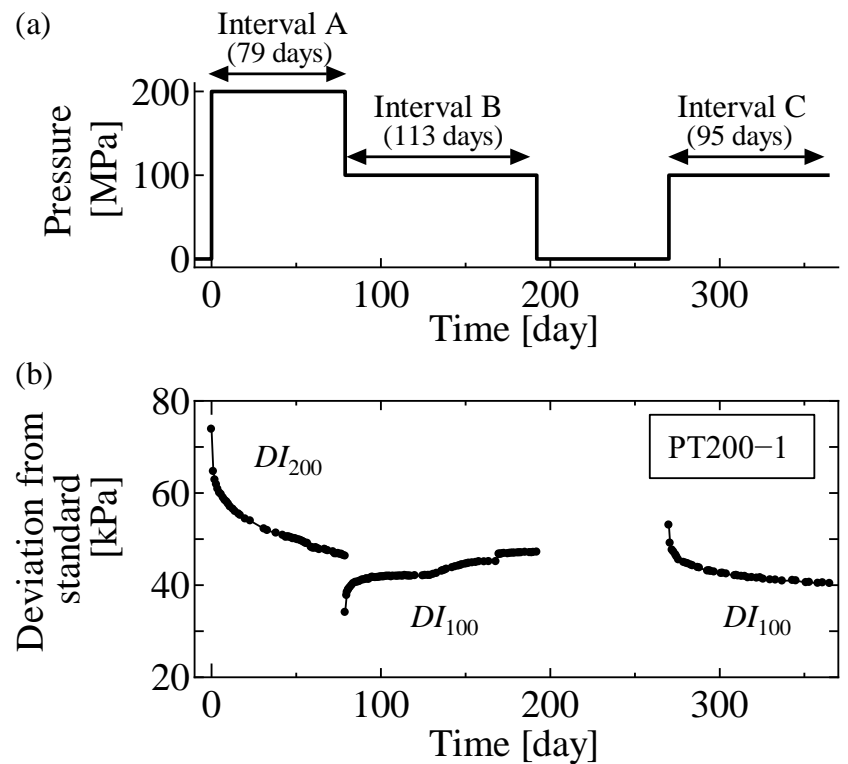

Figure 2. (a) History of pressure application to three pressure gauges PT2001, PT200-2, and PT200-3. (b) Variation in the deviation of pressure indications from the standard, $D l_{\mathrm{p}}$, for PT200-1. 
PT200-2, and PT200-3. The three gauges were firstly pressurised from atmospheric pressure to $200 \mathrm{MPa}$ and maintained at $200 \mathrm{MPa}$ for 79 days (Interval A). Then, the pressure was decreased from $200 \mathrm{MPa}$ to $100 \mathrm{MPa}$, which was maintained for 113 days (Interval B). On the 192th day, the pressure was released to atmospheric pressure. After the pressure release for 78 days, the gauges were pressurised to 100 $\mathrm{MPa}$ on the 270th day, which was then maintained for 95 days (Interval C). The calibration values of the three gauges were traced during the respective pressure applications. Figure 2(b) shows the calibration values $D I_{\mathrm{p}}$ of PT200-1 during the experiment. The calibration value varies with time differently according to the applied pressure and previous pressure condition.

Figure 3 compares the long-term drift of the three pressure gauges. Figure 3(a) shows the variations in the calibration values at $200 \mathrm{MPa}, D I_{200}$, with the elapsed time since the pressure application. We observed three different drift characteristics, mainly according to the difference in the sensing structures of the three gauges.

The calibration values for PT200-1 rapidly decreased immediately after the constant pressure application. The value decreased by $9.2 \mathrm{kPa}, 46$ parts per million (ppm) relative to 200 $\mathrm{MPa}$, in the first day. The amount of the variation is large compared with the short-time stability of the gauge and it reached almost half of the claimed accuracy. The change rate

(a) Interval A, $200 \mathrm{MPa}$

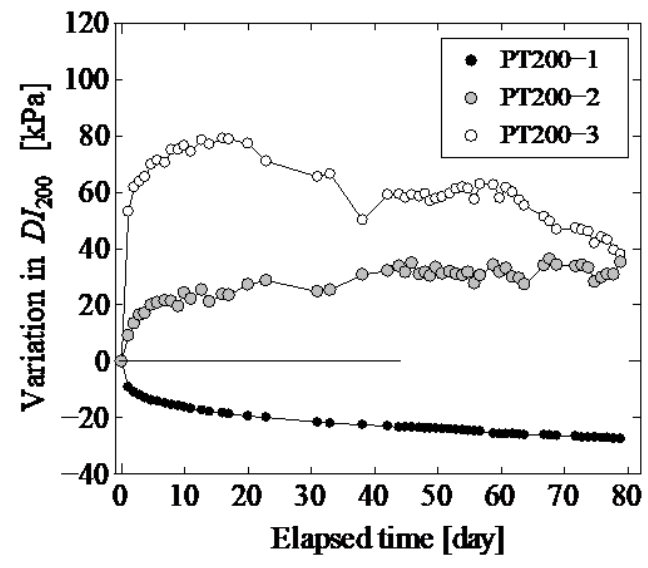

(b) Interval C, $100 \mathrm{MPa}$

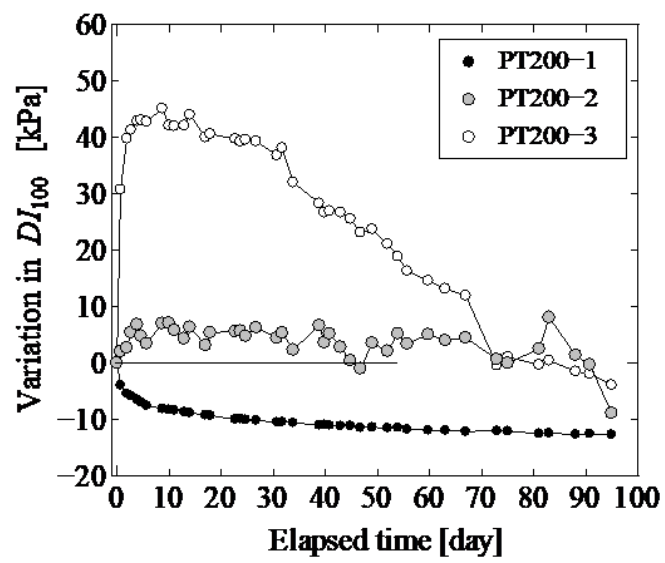

Figure 3. Variation in the calibration value according to elapsed time in days, under constant pressure application from atmospheric pressure: (a) $200 \mathrm{MPa}$ during Interval A in Figure 2(a), (b) $100 \mathrm{MPa}$ during Interval C in Figure 2(a). became smaller as time passed. The average change rate from the 20 th to the 80 th day was approximately $-0.13 \mathrm{kPa} /$ day, relatively $-0.65 \mathrm{ppm} /$ day. The total variation in $D I_{200}$ during the 80 days was $28 \mathrm{kPa}$, relatively $140 \mathrm{ppm}$, which exceeds the expanded $(k=2)$ uncertainty of $12.5 \mathrm{kPa}$ in the normal stepwise calibration. In contrast, the calibration values for PT200-2 increased with time. The variation in $D I_{200}$ during the 80 days was $40 \mathrm{kPa}$, relatively $200 \mathrm{ppm}$, which is larger than that for PT200-1. Most of the variation was made during the first 20 days. It should be noted that PT200-2 showed larger data scattering than the other gauges. The standard deviation of the obtained 18 indications was typically $5 \mathrm{kPa}$. The value seems almost constant within a data scattering after around the 40th day from the pressure application. PT200-3 showed a much larger and arch-shaped drift. At first, the calibration value rapidly increased by $60 \mathrm{kPa}$ in a few days, and it then turned to decrease at the rate of $-0.49 \mathrm{kPa} /$ day, relatively $2.4 \mathrm{ppm} /$ day, after the 20th day.

Figure 3(b) shows the variation in $D I_{100}$ according to time for the same three gauges during Interval $C$. The drift trends in $D I_{100}$ were similar to those in $D I_{200}$ for all the three pressure gauges. For PT200-1, DI 100 rapidly decreased at first, and then, the change rate became smaller. The total variation in $D I_{100}$ during 80 days was $13 \mathrm{kPa}$, which is almost half of the variation in $D I_{200}$. PT200-2 also showed the similar increasing drift trend, but the variation in $D I_{100}$ was relatively smaller and more scattered than that in $D I_{200}$. Similarly, PT200-3 showed an archshaped drift at $100 \mathrm{MPa}$. The amount of the first increase in $D I_{100}$ was about $45 \mathrm{kPa}$ during 15 days, which is almost half of the increase in $D I_{200}$. Both $D I_{200}$ and $D I_{100}$ turned to decrease after the first increase. From the above comparison, the gauges having the same sensing structure showed the similar drift trend when the pressure is increased from atmospheric pressure. The amount of the variation depends largely on the applied pressure.

The long-term drifts also depend on the previous pressure conditions. Figure 4 compares the drifts in $D I_{100}$ with different previous pressure conditions. As shown in Figure 2(a), the applied pressure was decreased from $200 \mathrm{MPa}$ to $100 \mathrm{MPa}$ at the beginning of Interval $\mathrm{B}$, while the pressure was increased from $0 \mathrm{MPa}$ (atmospheric pressure) to $100 \mathrm{MPa}$ at the beginning of Interval C. In Figure 4, the data in Interval B and Interval $\mathrm{C}$ is shown by closed and open circles, respectively.

In Figure 4(a), for PT200-1, DI 100 observed during Interval B and that during Interval $\mathrm{C}$ varied in the opposite direction with each other, although the amount of the variation during the 80 days was similar. The data during Interval B, denoted by closed circles, showed irregular drift behaviour. After becoming almost flat at around the 50th day, $D I_{100}$ started to increase again. This two-step trend was not observed when the gauge was pressurised from atmospheric pressure, and is thought to be due to the hysteresis effect. A discontinuous step by $2.5 \mathrm{kPa}$ was also observed on the 91st day, the reason for which remains unknown. For PT200-2, the two drifts were not symmetric. $D I_{100}$ in Interval B kept decreasing with time in larger amounts. For PT200-3, the direction of the drift in $D I_{100}$ during Interval B and that during Interval $\mathrm{C}$ were opposite to each other immediately after the pressure change. After 20 days, however, $D I_{100}$ both during Interval $\mathrm{B}$ and Interval $\mathrm{C}$ decreased over time at an almost constant rate. The average drift rates from the 30th day to 80th day, in Interval B and Interval C, were similar: The drift rates were $-0.71 \mathrm{kPa} /$ day in Interval $\mathrm{B}$ and $-0.75 \mathrm{kPa} /$ day in Interval $\mathrm{C}$. The previous pressure 
(a) PT200-1

- Interval B, from $200 \mathrm{MPa}$

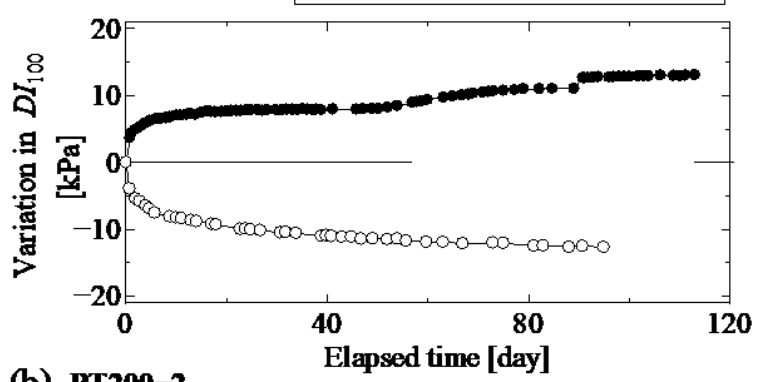

(b) PT200-2

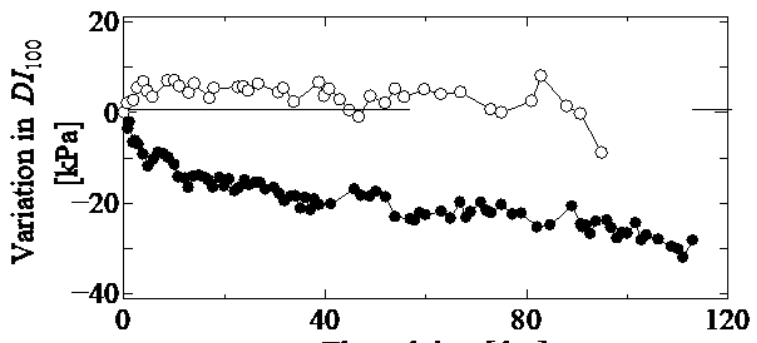

(c) PT200-3

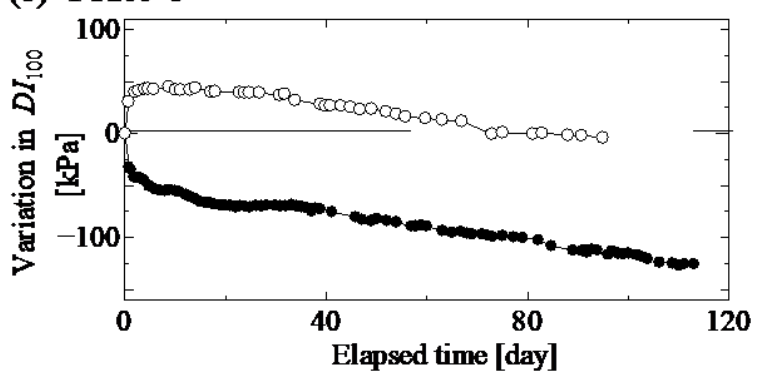

Figure 4. Variation in the calibration value according to elapsed time at 100 MPa for (a) PT200-1, (b) PT200-2, and (c) PT200-3. Data during Interval B and Interval $\mathrm{C}$ is shown by closed and open circles, respectively.

condition did not affect the drift behaviour of PT200-3 after a month, and a similar drift trend was noted.

As shown above, even under similar pressure conditions, the drift in the calibration values largely differs depending on the previous pressure condition. The effect of the previous condition depends on each pressure gauge. In some cases, preliminary application of a higher pressure makes the drift more complicated and makes correction of the drift difficult.

\subsection{Long-term drifts at measurement pressure and atmospheric pressure under constant pressure}

In the next experiment, we evaluated the variations in the calibration value with the elapsed time at $70 \mathrm{MPa}$ and $0 \mathrm{MPa}$ (atmospheric pressure) when the pressure of $70 \mathrm{MPa}$ was constantly applied to two pressure gauges PT70-1 and PT70-2. In this experiment, we intermittently released the applied pressure for a short time after the measurement at $70 \mathrm{MPa}$. The calibration value at atmospheric pressure $D I_{0}$ were then calculated as the deviation of the output from the reference value obtained by the reference atmospheric pressure gauge. This procedure was also explained in [13]. Figure 5 shows the variations in pressure gauge indications according to time after the pressure release. The horizontal axis shows the elapsed time after the pressure was decreased to atmospheric pressure, and oil overflowed from a release port. Figure 5(a) shows the indications of PT70-1 together with the variations in (a) PT70-1

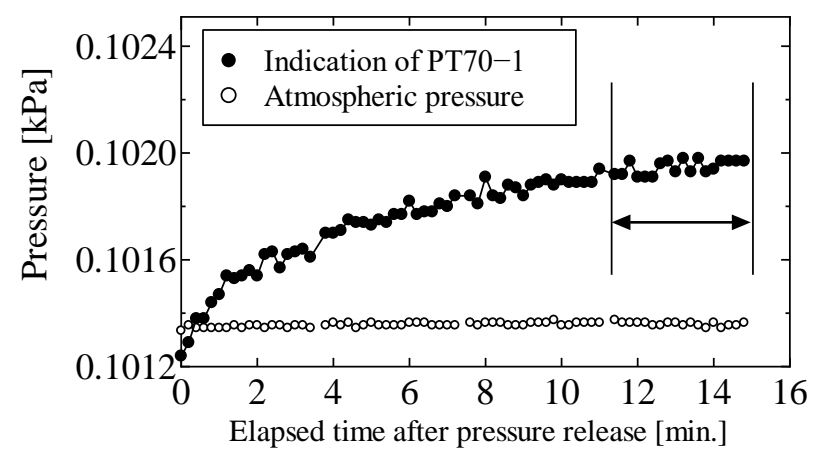

(b) PT70-2

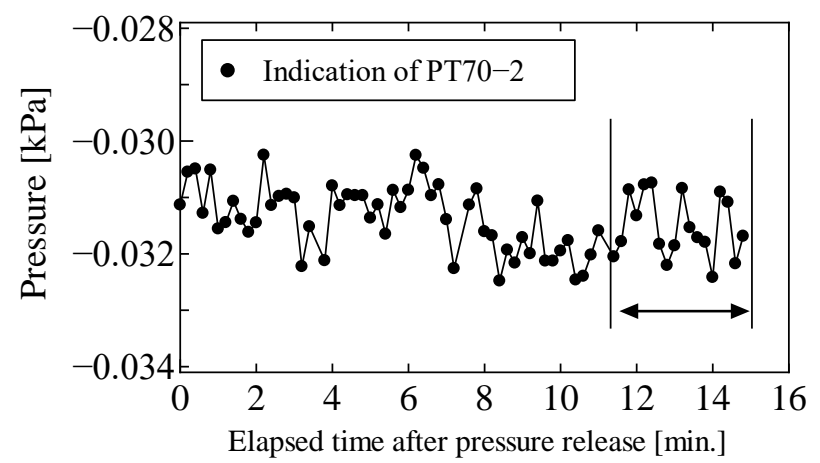

Figure 5. Variations in pressure gauge indications according to elapsed time after pressure release: (a) PT70-1 together with atmospheric pressure, (b) PT70-2.

atmospheric pressure. The indication of PT70-1 increased rapidly after the pressure release and approached a certain value, but atmospheric pressure was almost constant during that time. We used the averaged value during the last 3.5 minutes, as shown by a bidirectional arrow in Figure 5(a), and then obtained $D I_{0}$. The averaged values obtained during the same time interval were used for all the measurement points in order to avoid the effect of the measurement timing on the long-term drift evaluation. In contrast, the indications of PT70-2 in Figure 5(b) are almost constant within the data scattering, showing no time-dependent behaviour. After the measurement of $D I_{0}$, the test gauges were maintained at $70 \mathrm{MPa}$ again. Since the time span at atmospheric pressure was short, less than 20 minutes, these intermittent $D I_{0}$ measurements did not have effects on the results of the long-term drift.

Figure 6 shows the variations in the calibration value with the elapsed time at $70 \mathrm{MPa}, D I_{70}$, and at $0 \mathrm{MPa}, D I_{0}$. For PT70-1 in Figure 6(a), the drift trend of $D I_{70}$ is similar to $D I_{200}$ of PT200-1, showing a similarity in the drift behaviour for a similar sensing structure. In addition, the variations in $D I_{70}$ and $D I_{0}$ are almost parallel to each other. Although $D I_{70}$ decreased largely and nonlinearly immediately after the pressure application, the difference between $D I_{70}$ and $D I_{0}$ was maintained as constant during the 80-day experiment and only varied within approximately $0.2 \mathrm{kPa}$, relatively $3 \mathrm{ppm}$. In a previous study [13], a similar kind of gauge showed a similar result at $100 \mathrm{MPa}$. The drift of zero-reading is thought to be dominant for the drift under constant pressure application. Therefore, if the calibration values $D I_{0}$ at $0 \mathrm{MPa}$ (atmospheric pressure) were obtained during the constant pressure applications, most of the 
(a) PT70-1
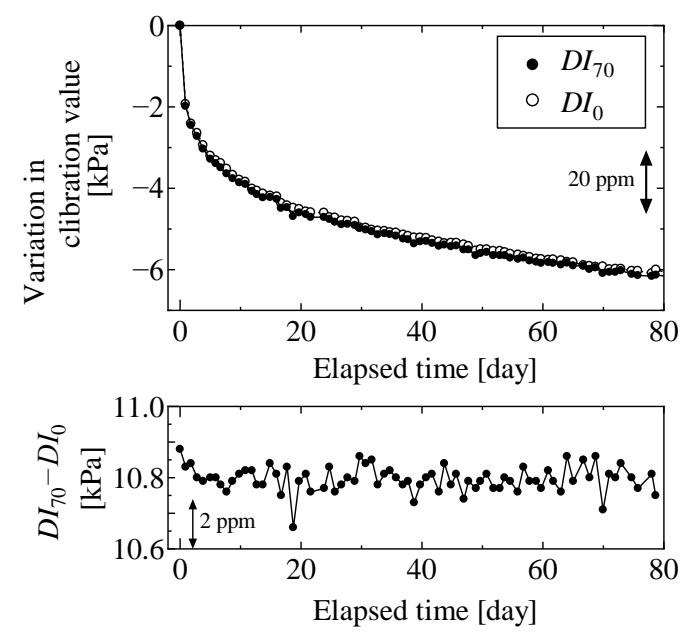

(b) PT70-2
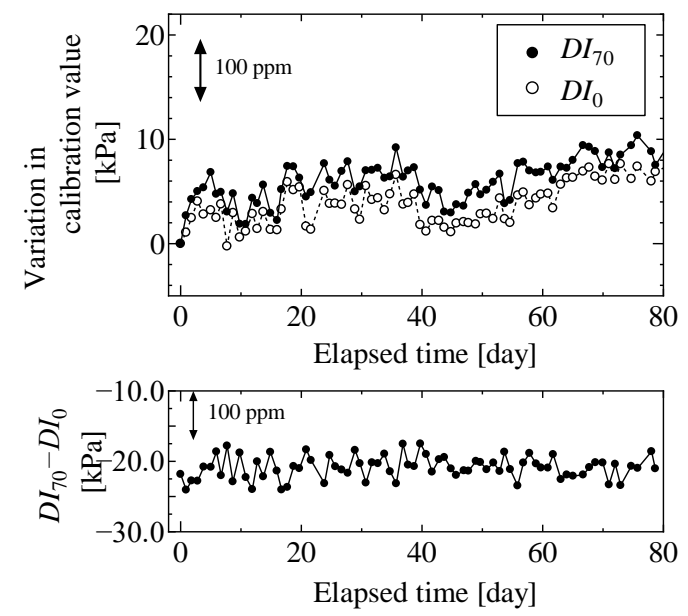

Figure 6. Variations in the calibration value according to elapsed time at 70 $\mathrm{MPa}(D / 70)$ and $0 \mathrm{MPa}(D / 0)$ : (a) PT70-1, (b) PT70-2. Variation in $(D / 70-D / 0)$ according to elapsed time is shown in the lower figures.

drift could be corrected by tracing $D I_{0}$ without calibration at the measurement pressure.

For PT70-2, in Figure 6(b), $D I_{70}$ slightly increased with time, which is also similar to the drift of PT200-2. In addition, the variation in $\left(D I_{70}-D I_{0}\right)$ with the elapsed time was maintained as constant, as shown in Figure 6(b), although the scattering in $D I_{70}$ and $D I_{0}$ is large.

\subsection{Discussion}

We observed three different drift characteristics depending on the kind of pressure gauges (sensing structures). Pressure gauges of the same model showed the same drift trend, if no pressure had been preliminarily applied to the gauges. To summarise the drift characteristics under the constant application of pressure, quartz Bourdon-type pressure transducers manufactured by Paroscientific Inc. showed a decreasing drift trend. The calibration value largely decreased at first, and then the drift rate became gradually smaller. In contrast, clamped thin-film gauges manufactured by WIKA showed an increasing trend. Foil strain gauges manufactured by HBM showed an arch-shaped drift trend. The calibration value first increased with time and then turned to decrease. It must be noted that although the drift trend of the gauges is reproducible, the amount of the drift depends on individual gauges, and even then, the drift is not quantitatively predictable only from the experiments for the selected samples. For precise pressure measurements at the actual site, experiments are necessary for respective gauges under similar pressure conditions for a time period similar to the actual measurements, although such experiments take a long time and are also costly.

One possible way to reduce and correct the long-term drift under pressurised conditions is preliminary pressure application. We observed significant and rapid variations in the calibration values immediately after the pressure application, but after a while, the drifts became smaller and showed almost constant variations. Thus, if the gauges are preliminarily pressurised at the measurement pressure, the first large drift during the actual usage could be suppressed, and the drift could be more easily corrected. This idea was also demonstrated in [13] for similar quartz Bourdon-type pressure transducers (Paroscientific, Inc.) at $100 \mathrm{MPa}$. From the first experiment in section 3.1, however, the preliminary applied pressure should be similar or very close to the measurement pressure, because the drift behaviour also depends on the preliminary pressure conditions. As demonstrated in Figure 4(a), irregular drift behaviour was observed for PT200-1, when the gauge was kept at $100 \mathrm{MPa}$ after being depressurised from $200 \mathrm{MPa}$. The application of different pressures may cause large hysteretic effects, leading to a more complicated drift.

If the drift of zero-reading is dominant for the drift under the constant application of pressure, most of the drift can be corrected by tracing $D I_{0}$, as shown in Figure 6, and the pressure can then be measured with a level of uncertainty similar to the normal calibrations. However, if the drift of the span is influential to the overall drift, the effectiveness of this method would be limited. We are continuously evaluating the effectiveness of this method for various gauges and under various conditions.

\section{CONCLUSIONS}

The long-term drifts of three kinds of pressure gauges under constant pressure applications have been investigated by tracing the calibration values totally for a year. In the first experiment, the drift behaviour of the three kinds of pressure gauges was compared at $200 \mathrm{MPa}$ and $100 \mathrm{MPa}$. The drift behaviour was completely different across the three gauges, mainly due to the difference in structures in the pressuresensing element. The long-term drift also depends largely on preliminary pressure conditions even under the same pressure application. In the second experiment at $70 \mathrm{MPa}$, the calibration values at atmospheric pressure intermittently obtained during the pressure application showed a similar trend to those at the measurement pressure.

Practical methods of evaluating and mitigating the long-term drift under constant pressure were discussed. Preliminary pressure application to the pressure gauge can reduce the amount of drift during actual usage. The preliminarily applied pressure should be similar or very close to the measurement pressure in order to avoid complicated hysteretic effects on the drift. In addition, the drift of zero-reading is the main contributory factor of the long-term drift in most cases; therefore, tracing the zero-reading during the pressure application would be highly useful for tracing and mitigating the drift. The findings and ideas will be applied to real problems in scientific and industrial fields, including pressure monitoring at seafloors. 


\section{REFERENCES}

[1] T. Kobata, Characterization of quartz Bourdon-type highpressure transducers, Metrologia 42 (2005) pp. S235-S238.

[2] J. Singh, A. Kumar, N.D. Sharma, A.K. Bandyopadhyay, Reliability and long term stability of a digital pressure gauge (DPG) used as a standard: A Case Study, MAPAN 26 (2011) pp. 115-124.

[3] I. Kocas, M. Bergoglio, An investigation of quartz type pressure transducer behavior under continuous pressure conditions and metrological characterization, Measurement 45 (2012) pp. 24862489.

[4] M. Kojima, T. Kobata, K. Fujii, Long-term stability and zero drift of digital barometric pressure gauges, Metrologia 52 (2015) pp. 262-266.

[5] H. Kajikawa, T. Kobata, Characterization and successive maintenance of transfer standard for APMP inter-laboratory comparison of hydraulic pressures, Proc. of the SICE Annual Conference, September 20-23, 2016, Tsukuba, Japan, pp. 782 785.

[6] A. P. Miiller, G. Cignolo, M. P. Fitzgerald, M. P. Perkin, Final report on key comparison CCM.P-K5 in Differential Pressure from $1 \mathrm{~Pa}$ to $1000 \mathrm{~Pa}$, Metrologia 39 (2002) Tech. Suppl. 07002.

[7] T. Kobata, A. K. Bandyopadhyay, K. Moore, A. A. E. Eltawil, S. Y. Woo, T. K. Chan, W. Jian, J. Man, N. N. Con, C. S. Fatt, Final report on key comparison APMP.M.P-K7 in hydraulic gauge pressure from $10 \mathrm{MPa}$ to $100 \mathrm{MPa}$, Metrologia 42 (2005) Tech. Suppl. 07006.
[8] Y. Ito, R. Hino, M. Kido, H. Fujimoto, Y. Osada, D. Inazu, Y. Ohta, T. Inuma, M. Ohzono, S. Miura, M. Mishina, K. Suzuki, T. Tsuji, J. Ashi, Episodic slow slip events in the Japan subduction zone before the 2011 Tohoku-Oki earthquake, Tectonophysics 600 (2013) pp. 14-26.

[9] L. M. Wallace, S. C. Webb, Y. Ito, K. Mochizuki, R. Hino, S. Henrys, S. Y. Schwartz, A. F. Sheehan, Slow slip near the trench at the Hikurangi Subduction zone, New Zealand, Science 352 (2016) pp.701-704.

[10] E. Araki, D. M. Saffer, A. J. Kopf, L. M. Wallace, T. Kimura Y. Machida, S. Ide, E. Davis, IODP Expedition 365 shipboard scientists, Recurring and triggered slow-slip events near the trench at the Nankai Trough subduction megathrust, Science 356 (2017) pp. 1157-1160

[11] A. Polster, M. Fabian, H. Villinger, 'Effective resolution and drift of Paroscientific pressure sensors derived from long-term seafloor measurements,' Geochem. Geophys. Geosyst. 10 (2009) Q08008.

[12] R. B. Wearn Jr and N. G. Larson, Measurements of the sensitivities and drift of Digiquartz pressure sensors, Deep Sea Research 29 (1982) pp.111-134

[13] H. Kajikawa, T. Kobata, Evaluation and correction for long-term drift of hydraulic pressure gauges monitoring stable and constant pressures, Measurement 134 (2019) pp.33-39. 